\title{
Impact of Diabetes Mellitus on Outcome of Patients Undergoing Carotid Artery Stenting: Insights From a Single Center Registry
}

\author{
Hitinder S. Gurm, ${ }^{1 *} \mathrm{MD}$, Vivek Rajagopal, ${ }^{2} \mathrm{MD}$, Ravish Sachar, ${ }^{3} \mathrm{MD}$, \\ Alex Abou-Chebl, ${ }^{2}$ MD, Samir R. Kapadia, ${ }^{2}$ MD, Chris Bajzer, ${ }^{2}$ MD, FAcc, \\ and Jay S. Yadav, ${ }^{4}$ MD, FACC
}

\begin{abstract}
Objective: To evaluate the impact of diabetic status on outcome of patients undergoing carotid artery stenting (CAS). Background: Diabetes has been demonstrated to be a strong predictor of adverse outcome in patients undergoing coronary revascularization. Its significance in predicting outcome of patients undergoing carotid interventions has not been ascertained. Methods: We evaluated the short-term outcomes of 833 patients who underwent CAS at our institution. The primary outcome of this analysis was $\mathbf{3 0}$ day incidence of stroke, myocardial infarction, and death. Results: Diabetes was present in 311 patients. Baseline characteristics were comparable between diabetics and nondiabetics except for the diabetics having a lower left ventricular ejection fraction, lower hemoglobin, and a higher body mass index at baseline. Further, they were more likely to have congestive heart failure and coronary artery disease. There was no difference in the incidence of stroke $(1.9 \%$ versus $2.7 \%$,$) , myocardial infarction$ (MI) $(2.6 \%$ versus $1.9 \%)$, death (3.9\% versus $2.5 \%)$, or the composite of death stroke or MI (6.8\% versus $5.9 \%)$ at 30 days between diabetics and nondiabetics. Similar results were seen when the analysis was restricted to patients treated with an emboli protection device. Diabetes was not a risk factor for adverse outcome after CAS after multivariate adjustment. Conclusion: Diabetics undergoing CAS are more likely to have associated co-morbidities. However despite this handicap, their short term outcome after CAS is similar to that of nondiabetics. 02007 Wiley-Liss, Inc.
\end{abstract}

Key words: diabetes; carotid artery stenting; emboli protection devices

\section{INTRODUCTION}

Diabetes is a major health problem with the numbers of diabetics increasing both in the United States and globally. Cardiovascular disease is the leading cause of mortality and morbidity in the diabetic population [1]. When compared with nondiabetics, diabetics have a worse outcome after cardiovascular therapeutic interventions [2-4]. Diabetes is a major risk factor for stroke [5] and diabetics make up 11\%-40\% of patients undergoing carotid endarterectomy (CEA) [4]. Further, diabetes appears to be a major predictor of adverse outcome in this population [6].

Recently carotid artery stenting (CAS) has emerged as a viable alternative to CEA [7]. The impact of diabetes on outcome of patients undergoing CAS remains unknown. We accordingly evaluated the short and long term outcome of diabetic patients undergoing CAS at our institution.
${ }^{1}$ Division of Cardiovascular Medicine, University of Michigan, Ann Arbor, Michigan

${ }^{2}$ Cleveland Clinic Foundation, Cleveland, Ohio

${ }^{3}$ Wake Heart and Vascular Associates, Raleigh, North Carolina

${ }^{4}$ Cleveland, Ohio

All work presented in this analysis was performed at the Cleveland Clinic Foundation.

*Correspondence to: Hitinder S. Gurm, MBBS, Division of Cardiovascular Medicine, University of Michigan, TC B1-226, 1500 E Medical Ctr Drive, Ann Arbor, MI 48109-0311, USA.

E-mail: hgurm@med.umich.edu

Received 19 September 2006; Revision accepted 26 October 2006

DOI $10.1002 / \mathrm{ccd} .21020$

Published online 8 February 2007 in Wiley InterScience (www. interscience.wiley.com). 
TABLE I. Baseline Characteristics of the Cohort by Presence and Absence of Diabetes

\begin{tabular}{|c|c|c|c|}
\hline Baseline characteristics & $\begin{array}{l}\text { Nondiabetics } \\
(n=522)\end{array}$ & $\begin{array}{l}\text { Diabetics } \\
(n=311)\end{array}$ & $P$ \\
\hline Age (yr) & $70.6 \pm 10.4$ & $70.4 \pm 8.9$ & 0.336 \\
\hline Ejection fraction (\%) & $44 \pm 15$ & $41 \pm 16$ & 0.033 \\
\hline Female & $194(37.2)$ & $96(31.1)$ & 0.083 \\
\hline Height $(\mathrm{cm})$ & $169 \pm 11$ & $170 \pm 11$ & 0.333 \\
\hline Weight $(\mathrm{kg})$ & $77.2 \pm 15.4$ & $84.8 \pm 17.1$ & $<0.001$ \\
\hline Body mass index $\left(\mathrm{kg} / \mathrm{m}^{2}\right)$ & $26.9 \pm 4.8$ & $29.4 \pm 5.3$ & $<0.001$ \\
\hline \multicolumn{4}{|l|}{ Vascular Risk Factors } \\
\hline History of hypertension & $425(81.6)$ & $274(88.4)$ & 0.011 \\
\hline History of smoking tobacco & $391(75.5)$ & $220(71.0)$ & 0.165 \\
\hline History of hyperlipidemia & $391(75.6)$ & $253(81.6)$ & 0.047 \\
\hline Family history of premature arteriosclerosis & $94(18.1)$ & $58(18.6)$ & 0.72 \\
\hline \multicolumn{4}{|l|}{ Comorbidities } \\
\hline History of coronary artery disease & $392(75.2)$ & $267(86.1)$ & $<0.001$ \\
\hline History of unstable angina & $69(13.2)$ & $57(18.3)$ & 0.057 \\
\hline History of prior MI & $188(37.8)$ & $160(53.7)$ & $<0.001$ \\
\hline History of COPD & $92(17.6)$ & $60(19.3)$ & 0.578 \\
\hline History of CHF & $87(19.4)$ & $96(35.6)$ & $<0.001$ \\
\hline History of PVD & $176(40.2)$ & $123(47.3)$ & 0.069 \\
\hline \multicolumn{4}{|l|}{ Laboratory values } \\
\hline Baseline creatinine (mg/dl) & $1.2 \pm 0.8$ & $1.3 \pm 1.0$ & 0.052 \\
\hline Baseline hemoglobin (g/dl) & $13.25 \pm 1.70$ & $12.81 \pm 1.78$ & 0.001 \\
\hline Platelet count, $(\mathrm{K} / \mu \mathrm{l})$ & $229 \pm 69$ & $224 \pm 75$ & 0.061 \\
\hline \multicolumn{4}{|l|}{ Procedural variables } \\
\hline Contralateral carotid occlusion & $71(13.7)$ & $47(15.4)$ & 0.537 \\
\hline History of prior radiation & $49(9.4)$ & $16(5.2)$ & 0.032 \\
\hline History of radical neck surgery & $26(5.0)$ & $10(3.2)$ & 0.291 \\
\hline Prior carotid endarterectomy & $132(26.7)$ & $56(18.7)$ & 0.010 \\
\hline CAS preopen heart surgery & $74(14.2)$ & $64(20.6)$ & 0.021 \\
\hline History of TIA within 6 months & $173(33.2)$ & $79(25.6)$ & 0.023 \\
\hline History of stroke & $137(26.2)$ & $95(30.7)$ & 0.174 \\
\hline History of Amarousis fugax & $45(8.7)$ & $15(4.8)$ & 0.052 \\
\hline Heparin dose(units) & $5466 \pm 1910$ & $5851 \pm 2093$ & 0.006 \\
\hline Peak activated clotting time (s) & $303 \pm 46$ & $298 \pm 46$ & 0.173 \\
\hline First direct systolic blood pressure $(\mathrm{mm} \mathrm{Hg})$ & $154 \pm 28$ & $157 \pm 27$ & 0.195 \\
\hline Emboli protection use & $341(65.3)$ & $219(70.4)$ & 0.147 \\
\hline Length of stay (in days) & $3.6 \pm 7.1$ & $3.9 \pm 6.6$ & 0.044 \\
\hline
\end{tabular}

Data are mean $\pm \mathrm{SD} ; n$, number of patients, values given in parenthesis indicate percentage.

\section{METHODS}

All patients undergoing CAS at our institution are followed in an institutional review board approved carotid stent registry. The details of this registry have been previously published $[8,9]$. Briefly, all patients are evaluated by a endovascular specialist and a neurologist prior to the procedure. Baseline demographic and clinical characteristics, interventional devices, procedural outcomes, and clinical complications are recorded on all patients.

All patients received aspirin, a thienopyridine (ticlopidine or clopidogrel), and intravenous unfractionated heparin. Majority of patients were also enrolled in different clinical trials or post-marketing registries of various stent platforms and emboli protection devices (EPDs). An ECG was obtained prior to and the day after the procedure. CK-MB was routinely ascertained 6-8 $\mathrm{hr}$ after the procedure, the morning after the procedure and in the event of suspected ischemia. All patients were evaluated by a neurologist prior to discharge. Stroke was defined as any focal nonconvulsive neurological deficit (corresponding to a vascular territory) persisting more than $24 \mathrm{hr}$. Outpatient follow-up was scheduled at $\sim 30$ days, 6 months, and annually thereafter. Mortality follow up on all patients was obtained using social security death index. Diabetes was defined by use of insulin, oral hypoglycemic agents, or patient report.

\section{Statistical Analysis}

Continuous variables are expressed as mean +/standard deviation while discrete variables are expressed as frequency counts and percentages. Difference in discrete variables between groups was determined using the chi square test, while the $t$ test and Mann-Whitney $\mathrm{U}$ test were used for continuous variables as appropriate. Binary logistic regression was used to calculate the adjusted and unadjusted odds of death, myocardial infarction 


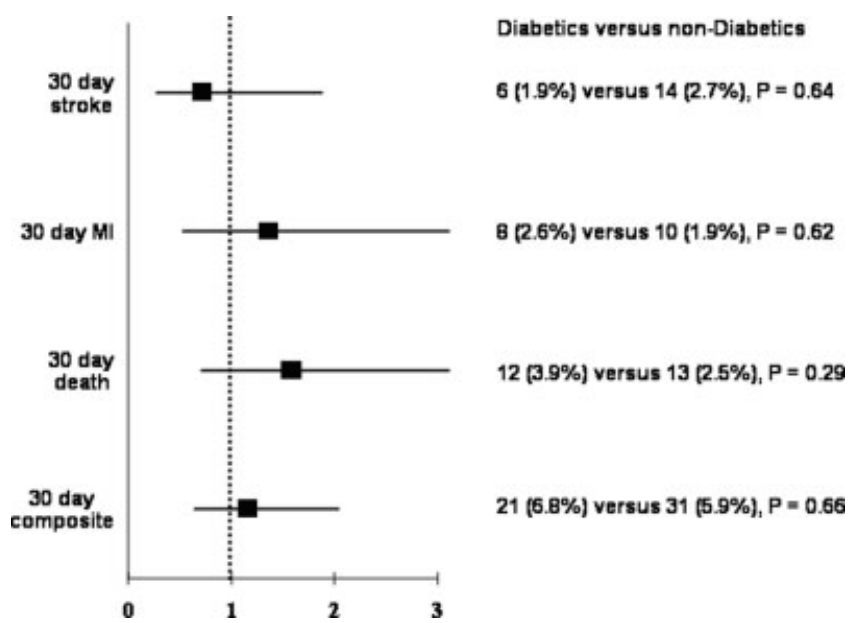

Fig. 1. The odds and incidence of major adverse events at $\mathbf{3 0}$ days by diabetes.

(MI), stroke or a composite of death, MI or stroke at 30 days. A logistic regression model was developed to assess for independent predictors of adverse outcome. Discriminating ability and goodness of fit were assessed using the $\mathrm{c}$ statistic and the Hosmer-Lemeshow test, respectively.

Long term survival was illustrated with KaplanMeier curves, and outcomes were compared using the log-rank test. A Cox regression model was developed to identify independent predictors of survival among patients undergoing carotid stenting.

\section{RESULTS}

Our cohort comprised of 833 patients that underwent their first CAS at our institution between February 1998 and August 2005. Diabetics made up 37\% (311) of the cohort. The baseline characteristics of the cohort by diabetic status are described in Table I. Diabetics were similar with respect to age and gender but were more likely to have coexistent coronary artery disease, prior MI, congestive heart failure, hypertension and dyslipidemia, a greater body mass index, and a lower hemoglobin. They were less likely to have had prior radiation therapy to the neck or have undergone prior CEA.

\section{Short Term Outcome}

The short term outcome of the cohort is described in Fig. 1. There was no difference in the incidence of death, stroke or MI or the composite of stroke, death or MI at 30 days between diabetics and nondiabetics. Similarly when patients treated with EPDs were evaluated separately, there was no difference in the short term outcome of diabetics and nondiabetics (Fig. 2).

The mode developed to predict 30 day MACE had good discriminating ability and goodness of fit (c statistic

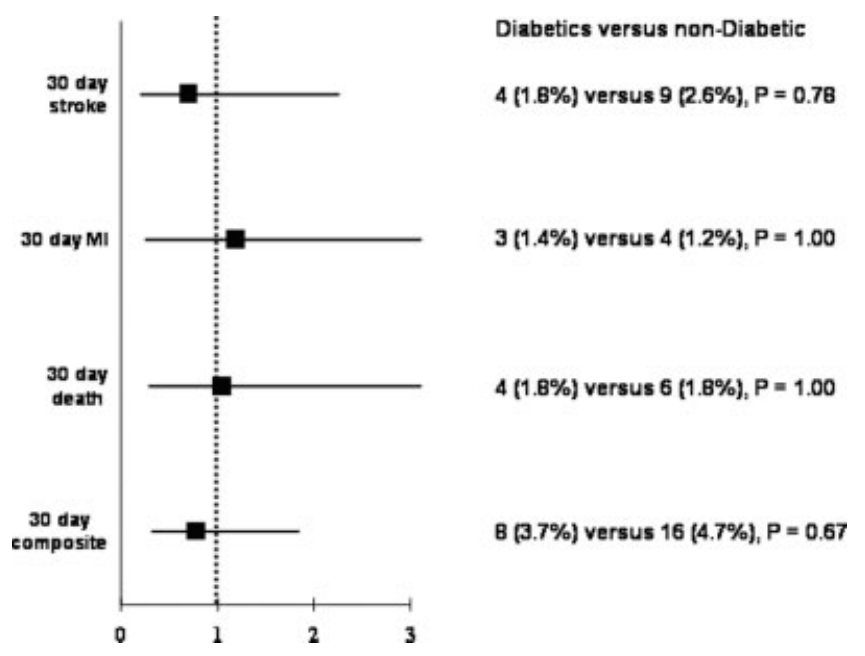

Fig. 2. The odds and incidence of major adverse events at $\mathbf{3 0}$ days by diabetes in patients treated with emboli protection devices.

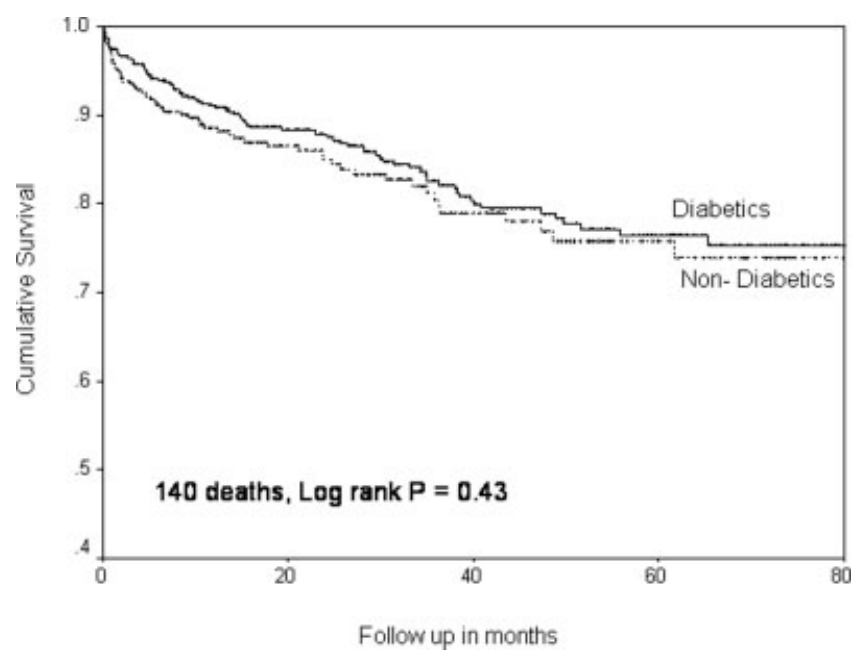

Fig. 3. The long term survival of the cohort based on diabetic status.

of 0.94, Hosmer-Lemeshow $P=0.92$ ) The independent significant predictors of 30 day death, stroke or MI were (in the order of importance) CAS performed prior to open heart surgery (OR 3.12), hematocrit (OR 0.93), EPD use (OR 0.45), and age (OR 1.001). When diabetes was forced into this model, it was not a significant predictor of events (OR $0.99,95 \%$, CI $0.52-$ $1.84, P=0.97)$.

The long term survival of the cohort is depicted in Fig. 3. There were a total of 140 deaths over a mean follow up period of 31.5 months. There was no difference in the survival of diabetics and nondiabetics in this cohort (Log-rank $P=0.43$ ). Similarly, diabetes was not an independent predictor of survival in this cohort. The independent predictors of long term sur- 
vival were younger age, absence of history of congestive heart failure, use of EPD, greater body mass index, higher systolic blood pressure at time of procedure, higher baseline hemoglobin, and lower baseline serum creatinine.

\section{DISCUSSION}

The key finding of our study is that when compared with nondiabetics, diabetics undergoing carotid stenting are not at any greater risk of peri-procedural complications (including death). These findings are important for many reasons. Carotid stenting has evolved from a nascent field to a mature technology and the number of procedures being performed worldwide is increasing [10]. Since diabetics make up a large proportion of patients undergoing CEA, the number of diabetics undergoing carotid stenting is expected to increase.

Previous data indicate that diabetics undergoing CEA probably have a similar to slightly increased risk of perioperative stroke but a higher risk of peri-procedural MI and death [4]. Data from the Swedish Vascular registry suggests that diabetes is an independent predictor of early death after CEA [11]. Other contemporary data also suggest that diabetics have a lower short and long term survival after CEA [12]. Similarly diabetes has been demonstrated to be an independent risk factor for peri-procedural MI [13]. In the recently published analysis of over 13,000 patients undergoing CEA, diabetic patients had a significantly higher risk of stroke $(2.1 \%$ versus $1.5 \%)$, death $(3.1 \%$ versus $1.6 \%)$, or MI (1.6\% versus $1 \%)$.

The reasons for the increased risk of peri-operative death and MI may relate to a greater prevalence of severe coronary artery disease among the diabetics undergoing CEA [14]. In our cohort, the prevalence of coronary artery disease was much higher in the diabetics and is concordant with data from the surgical literature [14].

Diabetics, in general have somewhat worse outcome after PCI and appear to derive a survival benefit from CABG [2,15-17]. The reasons for this poor outcome may relate to more diffuse disease, an inflamed vascular bed and an increased propensity to restenosis. There are many reasons why the same results may not apply to carotid arteries. The carotid arteries are large and the disease process is usually limited to the proximal internal carotid artery in both diabetics and nondiabetics. Further the markedly lower risk of perioperative MI with CAS may be particularly relevant to this population [7]. Indeed, the risk of MI in our population was exceedingly low. This finding is especially important since all patients at our institution are prospectively screened for occurrence of asymptomatic MI.

\section{Limitations}

Data on diabetic control and specific antidiabetic medications were not available in all patients and could not be incorporated into the analysis. Our study cohort relates to a selective patient population undergoing CAS in a tertiary care centre and selection bias cannot be excluded. It is possible that the nondiabetics in our population comprised of individuals with exceedingly aggressive atherosclerosis and the lack of difference in events may relate to a higher incidence of complications in nondiabetics. However, nondiabetics in our study were more likely to be undergoing the procedure for anatomic high risk while the diabetics were more likely to have medical (especially cardiac) high risk suggesting that this is unlikely. Further, the small number of events in the cohort prohibits multivariable adjustment for individual endpoints and also limits our ability to exclude a small but significant difference between the two groups. While our data set is large, the number of patients and events is still too small to determine if $6.8 \%$ event rate in diabetics is different than a $5.9 \%$ rate in nondiabetics. However, this low hazard also provides reassuring data on the safety and durability of the procedure.

\section{CONCLUSIONS}

Diabetics undergoing CAS do not appear to be at an increased risk of procedural complications. While direct comparative data is lacking, our findings suggest that CAS may be a viable alternate strategy for diabetic undergoing CEA. Further trials are warranted to directly test the two revascularization strategies in diabetic patients.

\section{REFERENCES}

1. Biondi-Zoccai GG, Abbate A, Liuzzo G, Biasucci LM. Atherothrombosis, inflammation, and diabetes. J Am Coll Cardiol 2003; 41:1071-7.

2. Gurm HS, Sarembock IJ, Kereiakes DJ, et al. Use of bivalirudin during percutaneous coronary intervention in patients with diabetes mellitus: An analysis from the randomized evaluation in percutaneous coronary intervention linking angiomax to reduced clinical events (REPLACE)-2 trial. J Am Coll Cardiol 2005; 45:1932-8.

3. Gurm HS, Lincoff AM, Lee D, et al. Outcome of acute ST-segment elevation myocardial infarction in diabetics treated with fibrinolytic or combination reduced fibrinolytic therapy and platelet glycoprotein IIb/IIIa inhibition: Lessons from the GUSTO V trial. J Am Coll Cardiol 2004;43:542-8.

4. Weiss JS, Sumpio BE. Review of prevalence and outcome of vascular disease in patients with diabetes mellitus. Eur J Vasc Endovasc Surg 2006;31:143-50.

5. Almdal T, Scharling H, Jensen JS, Vestergaard H. The independent effect of type 2 diabetes mellitus on ischemic heart disease, stroke, and death: A population-based study of 13,000 men and women with 20 years of follow-up. Arch Intern Med 2004; $164: 1422-6$. 
6. Stoner MC, Abbott WM, Wong DR, et al. Defining the high-risk patient for carotid endarterectomy: An analysis of the prospective national surgical quality improvement program database. J Vasc Surg 2006;43:285-295; discussion 295-6.

7. Yadav JS, Wholey MH, Kuntz RE, et al. Protected carotid-artery stenting versus endarterectomy in high-risk patients. N Engl J Med 2004;351:1493-501.

8. Gurm HS, Fathi R, Kapadia SR, et al. Impact of body mass index on outcome in patients undergoing carotid stenting. Am J Cardiol 2005;96:1743-5.

9. Saw J, Gurm HS, Fathi RB, et al. Effect of chronic kidney disease on outcomes after carotid artery stenting. Am J Cardiol 2004; 94:1093-6.

10. Wholey MH, Al-Mubarek N. Updated review of the global carotid artery stent registry. Catheter Cardiovasc Interv 2003;60: 259-66.

11. Kragsterman B, Logason K, Ahari A, et al. Risk factors for complications after carotid endarterectomy-A population-based study. Eur J Vasc Endovasc Surg 2004;28:98-103.

12. LaMuraglia GM, Brewster DC, Moncure AC, et al. Carotid endarterectomy at the millennium: What interventional therapy must match. Ann Surg 2004;240:535-44; discussion 544-6.
13. Aziz I, Lewis RJ, Baker JD, Virgilio C. Cardiac morbidity and mortality following carotid endarterectomy: The importance of diabetes and multiple Eagle risk factors. Ann Vasc Surg 2001;15: 243-6.

14. Hertzer NR, Young JR, Beven EG, et al. Coronary angiography in 506 patients with extracranial cerebrovascular disease. Arch Intern Med 1985;145:849-52.

15. BARI I. Comparison of coronary bypass surgery with angioplasty in patients with multivessel disease. The bypass angioplasty revascularization investigation (BARI) investigators. N Engl J Med 1996;335:217-25.

16. Mathew V, Frye RL, Lennon R, Barsness GW, Holmes DR, Jr. Comparison of survival after successful percutaneous coronary intervention of patients with diabetes mellitus receiving insulin versus those receiving only diet and/or oral hypoglycemic agents. Am J Cardiol 2004;93:399-403.

17. Mathew V, Gersh BJ, Williams BA, et al. Outcomes in patients with diabetes mellitus undergoing percutaneous coronary intervention in the current era: A report from the Prevention of REStenosis with Tranilast and its Outcomes (PRESTO) trial. Circulation 2004;109:476-80. 